\title{
¿HÉROE ESTRUCTURALISTA O ANOMALÍA POST-ESTRUCTURALISTA? DELEUZE COMO PENSADOR DE LA INDIVIDUACIÓN*
}

\author{
Alberto Toscano* \\ doi: 10.11144/Javeriana.uph37-74.heap
}

\section{RESUMEN}

Este artículo rastrea el vínculo entre la filosofía de la individuación de Deleuze, desarrollada en obras como Diferencia y repetición y Lógica del sentido, y la noción de estructura, alrededor de la cual se había organizado buena parte de la discusión epistemológica y ontológica de las ciencias y la filosofía francesas en la década de los sesenta. El autor no solamente señala la originalidad de la interpretación deleuziana del término estructura, - esta deja de corresponder a una invariante formal opuesta o ajena a la génesis de los individuos singulares y se transforma en un campo virtual de determinaciones preindividuales- sino que, además, expone los posibles límites de su uso en una filosofía de la diferencia en sí misma. La reconstrucción de esta apuesta teórica que pretendía hacer del "estructuralismo" una nueva filosofía trascendental permite evaluar qué tanto de esta idea de estructura sobrevive en la obra posterior de Deleuze y Guattari y hasta qué punto su filosofía transforma o desplaza el proyecto trazado en estos textos iniciales.

Palabras clave: estructura; Deleuze; individuación; ontogénesis; subjetividad

Traducción de Juan Fernando Mejía Mosquera, Pontificia Universidad Javeriana, Bogotá, Colombia.

Goldsmiths, University of London, Londres, Inglaterra.

Correo electrónico: a.toscano@gold.ac.uk

La versión original de este artículo fue presentada, con el mismo título, como ponencia en el congreso internacional Deleuze: una imagen del pensamiento, celebrado en la Pontificia Universidad Javeriana en agosto de 2005. Parte de este material fue utilizado para componer el último capítulo 


\title{
STRUCTURALIST HERO OR POST-STRUCTURALIST ANOMALY? DELEUZE AS A THINKER OF INDIVIDUATION
}

\begin{abstract}
This article traces the link between Deleuze's philosophy of individuation, developed in such works as Difference and Repetition and Logic of Sense, and the notion of structure, that articulated a good part of the epistemological and ontological discussions in French philosophy and science in the 1960s. Besides pointing out the originality of Deleuze's take on the term 'structure' - as it ceases to correspond to a formal constant that is opposed or indifferent to the genesis of singular individuals, and becomes a virtual field of pre-individual determinations-, the author presents the possible limits of its use in a philosophy of difference in itself. The reconstruction of this philosophical bet that wanted to turn "structuralism" into a new transcendental philosophy allows to assess how much of this idea of structure remains alive in Deleuze and Guattari's later works, and to what extent their philosophy transforms or displaces the project that had been formulated in those early books.
\end{abstract}

Keywords: structure; Deleuze; individuation; ontogenesis; subjectivity

del libro de A. Toscano The Theater of Production. Philosophy and individuation between Kant and Deleuze, 2006, Palgrave Macmillan. Reproducido con autorización de Palgrave Macmillan.

Para citar este artículo: Toscano, A. (2020). ¿Héroe estructuralista o anomalía post-estructuralista? Deleuze como pensador de la individuación. Universitas Philosophica, 37(74), 17-35. ISSN 01205323, ISSN en línea 2346-2426. doi: 10.11144/Javeriana.uph37-74.heap 
EN SU ARTículo Sobre el estructuralismo de 1967, Deleuze (2005f) había dado la bienvenida a la emergencia de "una nueva filosofía trascendental" (p. 223), aludiendo a un proyecto filosófico que lo vincularía a autores que van desde Althusser hasta Foucault ${ }^{1}$. Al tratar de aislar un común denominador detrás de los esfuerzos aparentemente dispares de estos contemporáneos, Deleuze se orientó a la búsqueda creativa de un esquema común. Lo identificó, por un lado, como compuesto por el aislamiento y la construcción de estructuras virtuales diferenciadas internamente o campos abstractos de relaciones y, por el otro, por medio de la tarea complementaria de rastrear las circunstancias de la actualización espaciotemporal de estas estructuras en un estado de cosas diferenciado o actual ${ }^{2}$.

Esta interpretación del estructuralismo, que se extendería en la teoría de las ideas de Diferencia y repetición, está evidentemente condicionada por el encuentro con la teoría de las dos clases de multiplicidades que aparece en los estudios sobre Bergson, aunque, como veremos, implica una suerte de cambio con respecto a las primeras formulaciones de la ontología de Deleuze. Una vez más, Deleuze aspira a esbozar una forma de determinación no-representacional, a saber, la diferencia interna, que no sucumba a una contemplación mística de lo indiferenciado ni presuponga ilegítimamente individualidades constituidas -sean estas sustancia o esencia, sujeto u objeto-. Las multiplicidades del estructuralismo son definidas allí como ideas virtuales. Estas son continuos determinables y sistemas diferenciados. Como escribe Deleuze (2002a), "una Idea es una multiplicidad continua y definida de $n$ dimensiones" (p. 277). Como sistema de relaciones diferenciales, estas estructuras virtuales están determinadas recíprocamente. En tanto estas relaciones implican la distribución de singularidades preindividuales que delinean el espacio y el tiempo implicados en la estructura, son

1 Aunque el ensayo en efecto anticipa mucho de Diferencia y repetición (1968/2002a) y Lógica del sentido (1969/2005g), fue publicado solamente en 1973, en un momento en que la valoración del estructuralismo por parte de Deleuze se había vuelto mucho menos efusiva.

2 Sobre la distinción entre diferenciación actual y diferenciación virtual, véase Deleuze, 2002a, pp. 314-315. 
completamente determinadas (Deleuze, 2005a, p. 231). Además, como anota, la estructura no tiene nada que ver con la forma:

la estructura no se define por la autonomía del todo, por el primado del todo con respecto a sus partes, por una Gestalt que se ejercería en lo real y en la percepción; la estructura, al contrario, se define por la naturaleza de ciertos elementos atómicos que pretenden dar cuenta, al mismo tiempo, de la formación de los todos y de la variación de sus partes (2005f, p. 226) ${ }^{3}$.

Por lo tanto, las estructuras no son meramente los medios para la formalización, sino que brindan, por el contrario, los elementos genéticos de una morfogénesis.

La peculiaridad de la reconstrucción del estructuralismo de Deleuze reside en esta consideración de las estructuras no como esencias inmateriales ni invariantes formales, sino como las bases preindividuales de la individuación. A diferencia de sus contemporáneos, Derrida y Adorno, por ejemplo, Deleuze parece no registrar ningún obstáculo ni contradicción en la relación entre estructura y génesis. En lugar de representar entidades abstractas a un grado de distancia de las vicisitudes de la ontogénesis, las estructuras como multiplicidades internas son a la vez estáticas

3 Véase también Deleuze, 2002a, donde, al distinguir la determinación que corresponde a la estructura virtual de la de las actualidades en términos de mereología, escribe que: "se debe distinguir con cuidado el objeto como completo y el objeto como entero. Lo completo es solo la parte ideal del objeto, que participa con las otras partes de objetos en la Idea (otras relaciones, otros puntos singulares), pero que no constituye nunca una integridad como tal. Lo que falta a la determinación completa es el conjunto de determinaciones propias de la existencia actual" (p. 315).

4 La posición de Deleuze al respecto choca con la de Piaget. Para este, el estructuralismo relativiza la relación entre forma y contenido, de tal manera que una forma siempre puede servir como el contenido de otra. Este anidar de las formas es insuficiente desde el punto de vista de una teoría de las multiplicidades que busca explicar la génesis de la forma a partir de elementos no formales, lo que Mil mesetas (Deleuze \& Guattari, 2002) va a definir como "funciones sin forma". Véase Piaget, 1968, pp. 17-32. Esta también es la razón por la que Deleuze, a diferencia de Piaget y Vuillemin, parece atribuir poca importancia a la relación entre el estructuralismo y la teoría algebráica de grupos formulada por Évariste Galois. La lectura que Badiou (2002) hace de Deleuze falla al no notar esta implicación recíproca de estructura y génesis. La pretensión de Badiou de que el estructuralismo solamente es un análisis de seres reales (l'étant) que parte de una descomposición discreta de entidades se pierde de los trazos ontológicos y mereológicos de las ideas como multiplicidades variables. Para una crítica de este aspecto de la lectura de Badiou y cómo se apoya en una confusión sobre el concepto de lo distinto-oscuro y lo claro-confuso de Deleuze, véase Simont, 2002, pp. 469-472. 
(o atemporales) y dinámicas (o genéticas). Es el concepto de multiplicidad lo que brinda la clave para la articulación entre estas dos determinaciones aparentemente incompatibles. Considerada en la mutua inmanencia de las relaciones diferenciales que la componen, la estructura es estática, impasivamente indiferente al espacio-tiempo mensurable y a las magnitudes discretas que caracterizan la actualidad individuada. Pero esta diferencia interna deviene genética tan pronto como la multiplicidad de la estructura es actualizada, dividida, "medida". La ontogénesis es, por tanto, definida como el paso de una clase de multiplicidad, la portadora de la diferencia interna, a otra, la enumerable y clasificable multiplicidad de los seres actuales. Deleuze define esta génesis como estática porque la estructura misma, aunque provee la razón suficiente de la actualidad, no es un factor causal. Para que ese fuera el caso, la estructura tendría, ella misma, que ser discretamente individuada. En otras palabras, necesitaría estar ya reducida de su estado virtual a un estado actual. En esta disyunción de causa y génesis se revela la crucial disimetría entre la estructura y sus encarnaciones, entre lo virtual y lo actual.

Esta disimetría, en la que la génesis tiene lugar desde una virtualidad preindividual hasta una actualidad individuada, nos permite entender por qué Deleuze considera el estructuralismo como una filosofía de lo "inesencial". En la medida en que el orden de la estructura implicado como idea virtual cambia de clase a través de la división y actualización, la estructura no puede ofrecer en manera alguna el Uno de un Múltiple actual. Mientras que su determinación (recíproca y completa) como diferencia interna -su mínima extensión y su máxima comprensión, en cierta forma- le permite una forma singular de individualidad, no puede darle a lo actual una forma de identidad en la cual esta última pueda reconocerse a sí misma. Por esto la estructura no puede responder a la pregunta “¿qué?", a la pregunta por la esencia, la sustancia o la universalidad. Por el contrario, Deleuze propone considerar la estructura como un universal concreto, tal que las cosas actuales puedan ser vistas como soluciones locales que significan las conexiones asignificantes e ideales implicadas en la constitución virtual de la primera. Por eso el "acompañamiento" en el pensamiento de estos procesos de realización o individuación es lo que define la práctica de Deleuze como método de dramatización (2005c).

Como no se nos permite ninguna subsunción bajo universales o identificación de sustancias bajo las condiciones de la disimetría que define la génesis 
estática, la única lógica de investigación que queda es la de los casos, la del movimiento "descendente" desde la estructura a sus soluciones y del movimiento complementario de "ascenso" desde las actualidades constituidas a las condiciones diferenciales de su génesis 5 . La consecuencia más radical de eliminar la identidad en la cuestión de la determinación es, por supuesto, que el pensamiento debe construir tanto los campos problemáticos de individuación como sus soluciones. Este parece ser el sentido en el cual hemos de entender la exigencia de que cambiemos la pregunta “iqué?” por preguntas como “¿cuántos?”, “¿cómo?” o “¿en qué casos?” (2005d, p. 171). Dado el devenir inminente, tanto como inmanente, que distingue a las multiplicidades continuas - de tal manera que su separación es necesariamente su transformación, o bien en el "pensamiento", o bien en el "ser"-, no podemos ni contemplar esencias ni identificar universales. En lugar de esto, debemos investigar los dinamismos que afectan el propio nivel ideal, ese mismo nivel al que nos aproximamos inicialmente como estático.

En Diferencia y repetición, Deleuze (2002a) se enfrenta a esta pregunta en términos de lo que, siguiendo la Teodicea de Leibniz (2013), denomina procedimiento de vice-dicción. Dado que la diferencia interna de la estructura como multiplicidad ideal no puede ni ser identificada como esencia, ni sometida a un principio que pueda brindarle consistencia desde fuera, el pensamiento solo puede alcanzar la estructura individuándola, actualizando aquellas conexiones que al nivel de la diferencia de la estructura en sí misma permanecieron puramente ideales, esto es, determinables. Partiendo de la revisión de la multiplicidad ideal misma, lo que Deleuze llama el campo inicial, el pensamiento procede, así, por medio de operaciones complementarias, especificando campos adjuntos y condensando singularidades ${ }^{6}$. Esto significa que el acto del pensamiento, lo que Deleuze llama el ejercicio propio o trascendente del pensamiento, consiste en

5 Aunque parece ubicar toda determinación en el nivel de lo actual y adscribe todo dinamismo y determinabilidad a un virtual concebido como Uno-Todo, Badiou (2002) nos ofrece una introducción muy estimulante a estos dos caminos del método. Véanse en particular sus capítulos 4 y 5 (pp. 65-95).

6 Sobre la fuente matemática de la noción de un campo adjunto, véase Vuillemin (1962), pp. 222-233. Discutimos la noción de una condensación de singularidades con respecto a la esquematización que Deleuze realiza de la filosofía de la individuación de Whitehead en Toscano (2006, capítulo 2, sección 3, pp. 70-78). 
(1) revisar la multiplicidad inicial de modo tal que pueda desplegarse su estructura implícita y conectarla con otros elementos dentro del continuo atemporal de ideas que brindan los ingredientes para la transformación; y (2) trazar la línea que puede unir, o condensar, las singularidades correspondientes a las relaciones recíprocas dentro de la idea. Como escribe Deleuze (2002a):

Cada idea tiene como dos caras que son el amor y la cólera: el amor, en la búsqueda de fragmentos, en la indeterminación progresiva y en el encadenamiento de los cuerpos ideales de adjunción; la cólera, en la condensación de singularidades que define, a fuerza de acontecimientos ideales, la acogida de una "situación revolucionaria", y hace estallar la idea en lo actual (p. 288).

La revisión de la multiplicidad estructural que realiza el pensamiento produce, entonces, la determinación ordinal y progresiva (Deleuze, 2002a, p. 317) de las relaciones que constituyen su diferencia interna, espacializando, temporalizando y por último actualizando la idea en el kairos de una "ocasión sublime" Dentro de lo que Deleuze (2002a) llama la facultad de pensamiento puro, esta es la coyuntura en la cual el problema de la individuación, entendido como el punto de convergencia entre estructura y génesis, es inseparable de una transformación del pensador: "¿y a qué estábamos consagrados, sino a problemas que exigen hasta la transformación de nuestro cuerpo y de nuestra lengua?” (p. 290), pregunta. En otras palabras, ¿en qué estamos interesados, si no es en aquellas experiencias de lo virtual como campo preindividual que, definiendo la tarea del empirismo trascendental, exigen peligrosas individuaciones del pensamiento y del pensador a la vez?

Estas abigarradas preguntas plantean grandes desafíos. El más difícil tiene que ver con la relación entre génesis y causa. Como anotamos, es entre estos conceptos que Deleuze elige inscribir la disimetría de la individuación. Como él mismo

7 Deleuze (2005f) describe la duración de la idea en términos de "una temporalidad interna, variable según aquello que se actualiza” (p. 234); sobre la espacialización inmanente de la idea, Deleuze $(2005 \mathrm{~g})$ escribe sobre las singularidades que "se distribuyen en un campo propiamente problemático y sobrevienen en este campo como acontecimientos topológicos a los que no está ligada ninguna dirección" (p. 138). Para un tratamiento innovador, si bien algo unidimensional, de estos dos aspectos de la teoría deleuziana de la individuación a la luz de los modelos que propone la ciencia contemporánea, véanse los capítulos 2 y 3 de DeLanda, 2002. 
afirma enfáticamente en uno de sus seminarios sobre Leibniz y Whitehead (Deleuze, 2006): "una génesis no es causal" (pp. 255) ${ }^{8}$. Lo que hace que este asunto sea tan delicado es la pregunta de a cuál de los dos procedimientos (la génesis $o$ la causalidad) ha de concederse la prioridad al nivel de una teoría de la individuación y, además, cómo debe articularse esta diferencia en primer lugar. Esto es especialmente así en Lógica del sentido (Deleuze, 2005g), donde nos enfrentamos a la correlación no paralela o síntesis disyuntiva de una génesis no causal - en cuanto el sentido es un efecto impasible o un acontecimiento a un grado de separación de sus actualizaciones- y una causalidad no genética, por la cual la causalidad corporal determinaría un régimen intensivo despojado de cualquier explicación trascendental. Lejos de revelar una falla oculta en el corazón de la empresa de Deleuze, este problema se usa para extraer las paradojas a partir de las cuales está tejida Lógica del sentido, por tanto, situando de forma característica la vitalidad de la diferencia en el corazón de una heterogeneidad aparentemente incomposible. Por ende, el sentido mismo constituye la superficie bifronte (Janusheaded surface) que sintetiza estos dos sistemas desconectados (del cuerpo y las ideas) y permite su comunicación.

Deleuze (2005g) pregunta: “¿Cómo mantener a la vez que el sentido produce incluso los estados de cosas en los que se encarna, y que es producido por estos estados de cosas, acciones y pasiones de los cuerpos (inmaculada concepción)?” (p. 158). Vale la pena citar su respuesta en extenso:

La misma idea de génesis estática disipa la contradicción. Cuando decimos que los cuerpos y sus mezclas producen el sentido, no es en virtud de una individuación que lo presupondría. La individuación en los cuerpos, la medida en sus mezclas, el juego de las personas y los conceptos en sus variaciones, toda esta ordenación supone el sentido y el campo neutro, preindividual e impersonal en el que se despliega. Así pues, es de otro modo como el sentido es producido por los cuerpos. Se trata esta vez de los cuerpos tomados en su profundidad indiferenciada, en su pulsación sin medida. Y esta profundidad

8 De la misma manera, en su artículo sobre Hume (Deleuze, 2005e): "el problema no son las causas, sino el funcionamiento de las relaciones como efectos de esas causas, y las condiciones prácticas de ese funcionamiento" (p. 215). En el ensayo sobre Bergson de 1956 (Deleuze, 2005b) también comenta: "lo que explica la cosa misma es su diferencia, no sus causas" (p. 69). 
actúa de una manera original: por su poder de organizar superficies, de envolverse en superficies (p. 158).

Sin embargo, lo que permite en última instancia esta "síntesis heterogénea de la condición en una figura autónoma, que reúna en ella la neutralidad y la potencia genética" es, como nota Deleuze (2005g, p. 158), una instancia no condicionada. La comunicación de lo diferente con lo diferente -el sistema de los cuerpos y el sistema de las proposiciones, en este caso- no es un procedimiento que pueda ser cualificado por la misma inmanencia que caracterizaba la relacionalidad transductiva de Simondon. O, en su lugar, lo que Deleuze enfrenta en el despliegue de las consecuencias del estructuralismo como filosofía de la diferencia interna es la necesidad -más allá de las ideas-multiplicidades como portadoras de diferencia interna- de una instancia especulativa que permita la comunicación productiva entre la idealidad y la actualidad, las proposiciones y los cuerpos. Puede sostenerse que es para estar en guardia contra el retorno de la identidad y la semejanza, de la catastrófica teoría de la individuación específica que subtiende la filosofía de la representación, que Deleuze introduce su teoría del precursor oscuro o lo dispar (en Diferencia y repetición) y de la cuasi-causa u objeto aleatorio (en Lógica del sentido) ${ }^{9}$.

Es así como, en el vértice de la estructura, encontramos el problema de la diferencia en sí misma; la postulación de esta entidad paradójica que, mientras "falta a su propia identidad, falta a su propia semejanza, falta a su propio equilibrio, falta a su propio origen” (Deleuze, 2005g, p. 71) ${ }^{10}$, es, sin embargo, la que asegura la comunicación de las series, lo que impide que el momento necesario

9 Es importante notar que Deleuze (2002a) introduce este concepto en Diferencia y repetición a través de una secuencia de preguntas que ponen de manifiesto las posibles amenazas que podría plantear el recurso a este precursor sombrío para su teoría de la determinación a través de las ideas virtuales, de los dinamismos espaciotemporales intensivos y de sus producciones actuales: "ies en realidad la diferencia lo que relaciona lo diferente con lo diferente en estos sistemas intensivos? ¿La diferencia de diferencia relaciona la diferencia consigo misma sin otro intermediario? Cuando hablamos de una puesta en comunicación de series heterogéneas, de un acoplamiento y de una resonancia, ¿no es acaso con la condición de un mínimo de semejanza entre las series, y de una identidad en el agente que opera la comunicación?” (p. 186).

10 «Elle manque à sa propre identité, elle manque à sa propre ressemblance, elle manque à son propre équilibre, elle manque à sa propre origine ». 
de postular las diferencias de clase "degenere" en una mera distinción categorial (p. 71). De nuevo, el imperativo filosófico parece ser el de salvaguardar la diferencia interna (o individuante): ya no más como una noción individual, ni como la multiplicidad virtual de una idea, sino como tal $^{11}$. Sin embargo, es muy difícil ver cómo este diferenciador, aunque esté privado de toda identidad (selfidentity), puede abstenerse de constituir un principio estructural totalizador del cual dependerían, a su vez, las diferencias mismas. En otras palabras: una vez la comunicación entre series exige un intermediario paradójico, ¿̨cómo puede detenerse el deslizamiento hacia un último Diferenciador, como puro principio de producción anárquica?

Mientras Deleuze, a la luz de la teoría de la multiplicidad, nota frecuentemente que el Uno y lo Múltiple han de ser ellos mismos redescritos como clases determinadas de multiplicidad (2002b p. 88), no está claro cómo este objeto virtual $($ objeto $=\mathrm{x})$, este lugar vacio que está en todas partes y en ninguna a la vez y garantiza la comunicación de diferencias con diferencias, multiplicidades con multiplicidades, pueda él mismo ser considerado como una multiplicidad. Este es especialmente el caso si consideramos que, en su movimiento infinito a través de todos los sistemas de diferencia, aparece como desprovisto en sí mismo de dimensiones y singularidades. Este carácter estrictamente excepcional de la instancia que Deleuze ha introducido en Lógica del sentido como la "paradoja de Lacan” y que es definido en Diferencia y repetición como el diferenciador, aparece para restablecer la mismísima economía principal que la crítica de Simondon a los modelos de individuación clásicos y modernos había intentado suspender. Aunque no es un principio de individuación (tal vez tiene más de principio de anarquía, como el que Reiner Schürman [1990] extrae del último Heidegger), la síntesis disyuntiva que opera entre el sistema de la génesis ideal y el orden de las causas corporales, tanto como entre los sistemas diferenciales mismos, efectivamente parece coronarlo como lo incondicionado de cualquier posible individuación, aunque esté en el paradójico papel de un principio que podría socavar (unground) a todos los otros. La pregunta que se yergue en tal coyuntura es esta: ‘acaso los procesos "anómalos" de individuación -que emergen

11 "Lo importante, para el en-sí, es que, pequeña o grande, la diferencia sea interna" (Deleuze, 2002a, p. 188). 
de las heterogeneidades en los mecanismos de producción, de la comunicación generativa de lo diferente con lo diferente- exigen la garantía de un principio, no importa lo paradójico que este principio pueda ser? ¿Es la hegemonía de la filosofía de la identidad y la representación tal que su dispositivo de buen sentido y de sentido común solamente puede ser minado por la afirmación del caosmos de la diferencia como principio?

En la reconstrucción de Deleuze del estructuralismo como filosofía de la diferencia basada en los conceptos de multiplicidad y génesis estática, hemos encontrado ya una potente contribución al desarrollo de una filosofía trascendental (un materialismo trascendental) del ser preindividual, concebida como explicación de la determinación y determinabilidad de un real no-empirico, y de su función en el proceso de ontogénesis. Probablemente, el defecto de un enfoque semejante es que, salvo por la intervención sublime de un pensador dionisía$\mathrm{co}$, no delinea suficientemente los devenires que cubren el campo trascendental mismo, o aquellos que explican el sentido en el cual este campo es él mismo el lugar y no solamente la fuente o la cuasi-causa de las síntesis y variaciones. Además, al establecer una disyunción entre regímenes de individuación y al articular la demanda de que el campo trascendental no colapse en una distinción categorial, este momento estructuralista en la filosofía de la diferencia de Deleuze lo obliga a recurrir a un operador de heterogénesis (el diferenciador), que finalmente traiciona la promesa de la teoría de la multiplicidad, a saber, que la comunicación generativa de las diferencias podría ofrecer la clave para una filosofía de la individuación purgada de principios.

Sin embargo, de esto se sigue que la tesis "estructuralista" de la entidad paradójica sea, en nuestra opinión, un componente mucho menos necesario de Diferencia y repetición que de Lógica del sentido. La disyunción entre lo virtual y lo actual en aquel es, de hecho, una disyunción interna a, y generada por, los procesos mismos de ontogénesis. Considerada en términos de producción de lo discreto a partir de multiplicidades continuas, del movimiento a partir de singularidades preindividuales y de las relaciones recíprocas implicadas a un orden de individualidad explicable taxonómicamente, no parece requerir la misma solución que el singular problema de la relación entre los cuerpos y las proposiciones, planteado por Deleuze a propósito del acontecimiento del sentido. Fue solamente en Lógica del sentido (2005g) donde la noción de cuasi-causa apareció 
como una propuesta más consecuente y en realidad más necesaria. Sin embargo, si hemos de considerar los recursos conceptuales de Deleuze para una ontología de la individuación anómala, no podemos contentarnos con lo que yo llamaría la suficiencia de lo virtual, esto es, con la noción de que la asimetría de la ontogénesis implica que su proceso es unilateral, enteramente dirigido por lo virtual como su polo creativo. Aunque esta interpretación tiene muchas virtudes, desde el punto de vista del problema de la individuación, permanecer al nivel de una completa oposición entre diferenciación virtual y diferenciación actual parecería sugerir que la actualización no es otra cosa que la encarnación de una forma preconstituida. La filosofía de la diferencia solo confronta realmente el problema de la individuación cuando el movimiento de la diferencia interna es definido como una "indi-difereniación" (Deleuze, 2002a, p. 368), es decir, como proceso que requiere la dramatización de la multiplicidad interna en sistemas intensivos y dinamismos espaciotemporales. Es en esta faceta del pensamiento de Deleuze que su contribución a la filosofía de la individuación se vuelve más evidente. Es también, significativamente, ese aspecto de su enfoque de la cuestión de la diferencia interna lo que su posterior repudio de ciertas exigencias del estructuralismo deja esencialmente intacto.

En MIL MESETAS, Deleuze y Guattari (2002) distinguen dos enfoques de la ontología trascendental de la individuación, por medio de la diferencia entre el plano de organización (plan d'organisation) y el plano de inmanencia (plan dimmanence):

Desde este punto de vista, se dirá: veis perfectamente la diferencia entre los dos tipos de proposiciones siguientes, 1) unas formas se desarrollan, unos sujetos se forman, como función de un plan que solo puede inferirse ( $\mathrm{plan}[\mathrm{o}]$ de organización desarrollo); 2) tan solo hay velocidades y lentitudes entre elementos no formados, y afectos entre potencias no subjetivadas, como función de un plano que está necesariamente dado al mismo tiempo que aquello a lo que da lugar (plano de consistencia o de composición) (p. 270).

Es sobre la base de esta distinción que Deleuze y Guattari tratan de oponer su interés espinocista por la composición a la preocupación por la organización que 
identifican como el pecado original del estructuralismo. Al rechazar las ontologías organizacionales de la individuación en razón de sus deducciones genéticas y estructurales de la individualidad realizadas a partir de bases predeterminadas y trascendentes, ofrecen como alternativa otro modo de individuación, el cual, en homenaje a Escoto, denominan haecceidad.

Mientras que mucha de la terminología se ha transformado y el anterior intento de Deleuze de remodelar el estructuralismo como filosofía de la ontogénesis parece haber sido abandonado o incluso repudiado, esta última posición se encuentra todavía fuertemente determinada por las tesis ontológicas concernientes a los tipos de multiplicidad. Una haecceidad es, de hecho, definida aquí como una individuación que, esquivando la universalidad de las formas o la abstracción de las estructuras, compone directamente multiplicidades "planas" (flat) unas con otras. El terreno parece haber cambiado considerablemente con respecto a la preocupación anterior por las condiciones de realización -una preocupación que parecía permitir cierta continuidad con las explicaciones naturalizadas o materialistas de la ontogénesis-. Las individuaciones que Deleuze y Guattari ponen en primer plano en Mil mesetas (2002) no son del tipo que engendra individuos; por el contrario, atraviesan individuos ya constituidos, arrastrándolos a devenires impersonales, composiciones de una multiplicidad con otra: "una estación, un invierno, una hora, una fecha tienen una individualidad perfecta a la que no le falta nada, aunque esta individualidad es diferente de la de una cosa o un sujeto" (p. 264). Es como si, en lugar de reconfigurar el dominio de la producción trascendental desde adentro, al revelar la anomalía y la heterogeneidad que yacen bajo lo individuado, Deleuze y Guattari (2002) estuvieran abriendo una dimensión al lado de la de los seres constituidos, un mundo fugitivo de intensidades puras, alianzas y transformaciones -un mundo que ya no es más la razón suficiente de la diferencia sino su construcción y experiencia-: "aquí no hay en modo alguno formas o desarrollos de formas; ni sujetos y formación de sujetos. No hay ni estructura, ni génesis” (p. 269).

Mientras que en cierta forma esto lleva a demostrar que la idea del estructuralismo como una "nueva filosofía trascendental" ha sido dejada atrás en buena medida, puede también entenderse como la representación de un desplazamiento de algunos de los temas característicos de la obra de Deleuze al final de los años sesenta. Esto es especialmente claro si consideramos que lo que distingue el 
tipo de individuación (la multiplicidad) que Deleuze y Guattari llaman haecceidad no es otra cosa que aquello que en Diferencia y repetición habían identificado como dinamismos espaciotemporales, en otras palabras, "agitaciones de espacio, excavaciones de tiempo, pura síntesis de velocidades, direcciones y ritmos” (Deleuze, 2005c, p. 127) ${ }^{12}$. Una haecceidad, de hecho,

se define por una latitud y una longitud: es decir, el conjunto de los elementos materiales que le pertenecen bajo tales relaciones dadas de movimiento y de reposo, velocidad y lentitud (longitud); el conjunto de los afectos intensivos de los que es capaz, bajo tal poder o grado de potencia (latitud) (Deleuze \& Guattari, 2002, p. 264)

De hecho, a través del concepto de intensidad Diferencia y repetición se había aproximado al papel de las "velocidades y lentitudes" en la individuación, al presentar los dinamismos espaciotemporales como las operaciones que dramatizaban las ideas estructurales y efectuaban la comunicación de intensidades heterogéneas, constituyendo lo que Deleuze (2002a), siguiendo a Simondon, había llamado sistemas señal-signo. En Diferencia y repetición, como en los ensayos sobre el estructuralismo y la dramatización que lo preceden inmediatamente, la teoría de la individuación fue concebida para dar cuenta de la manera en que las diferencias implicadas en la estructura, junto con la disparidad que marca la intensidad, podrían dar cuenta del paso de la heterogeneidad y la diferencia interna a la clase de mundo explicado que está disponible para las operaciones de representación. El movimiento mismo de la diferencia interna a la externa encontró en estas operaciones puras a sus "agentes", los factores que permiten el paso de la impasividad de la estructura a la intensidad de los sistemas ${ }^{13}$, y de estos sistemas o campos de individuación al ámbito entrópico y extensivo de la representación.

12 Véase también Paradis, 1995, para una interpretación de Deleuze en que la velocidad es “una pura cualidad anterior a cualquier par de espacio y tiempo" (p. 14).

13 Esta es la definición de Deleuze (2005c) de los sistemas intensivos -su dependencia del pensamiento y la terminología de Simondon es considerable-: "si todo sistema es un campo intensivo de individuación construido por series limítrofes heterogéneas o dispares, la comunicación de las series, bajo la acción del precursor oscuro, produce fenómenos de acoplamiento entre las series, de resonancia interna en el sistema, de movimiento forzado al modo de una amplitud que desborda las propias series básicas" (p. 131). 
Sin la determinación que brindan estos factores de individuación, la génesis asimétrica que se mueve de las estructuras virtuales a los individuos constituidos sería no solamente estática, sino puramente ideal, o incluso idealista. Es por esto que Deleuze (2002a) - fiel al proyecto de Simondon de una teoría de las operaciones a la base de la ontogénesis- piensa que los dinamismos espaciotemporales

son precisamente dramas [...]. Estos crean, trazan un espacio correspondiente a las relaciones diferenciales y a las singularidades por actualizar [...]. El mundo es un huevo, pero el huevo es él mismo un teatro: un teatro de puesta en escena donde los papeles tienen más importancia que los actores, los espacios que los papeles y las ideas que los espacios (pp. 325-326).

En esta consideración de la individuación espaciotemporal, Deleuze (2002a) demuestra que cualquier explicación de la génesis real, la cual comienza desde "lo Desigual en sí, la disparidad tal como se comprende en la diferencia de intensidad como diferencia" -esto es, cualquier explicación de la individuación anómala- debe delinear las operaciones de individuación, tal como ellas determinan la división y recomposición de multiplicidades intensivas (p. 334).

La importancia de la teoría de la individuación como dramatización para el pensamiento de Deleuze como un todo está probada definitivamente por el hecho de que, cuando decide abandonar el proyecto estructuralista de una nueva filosofía trascendental, lo hace por medio de la adscripción de la primacía en sentido ontológico y ético a aquellos mismos dinamismos espaciotemporales a través de los cuales había aplicado la teoría de la individuación de Simondon a la problemática de la diferencia interna. En Mil mesetas (Deleuze \& Guattari, 2002), con el intento de pensar las multiplicidades intensivas y sus relaciones dispares (composiciones, agenciamientos, devenires, etc.) sobre un plano de inmanencia, es como si Deleuze y Guattari hubiesen generalizado la ontología de la individuación anómala tal como lo habrían hecho con cualquier determinación trascendente o con cualquier suficiencia. Las operaciones dinámicas, a través de las que se comunican y devienen las multiplicidades, ya no organizan ni encarnan nada más allá de las relaciones inmanentes entre sus factores individuantes ${ }^{14}$.

14 Sin embargo, en la teoría de la individuación de Diferencia y repetición (Deleuze, 2002a), "las ideas dominan” (tienen más importancia que) y, mientras tal vez no sean suficientes, en el análisis 
$\mathrm{Al}$ entregarle la individuación de todo el régimen de multiplicidades intensivas a "las velocidades y afectos" de las haecceidades, Deleuze y Guattari no están haciendo otra cosa que hacer el drama del ser enteramente inmanente. La individuación anómala -individuación de y desde lo Desigual, una integración cuyos únicos materiales son intensidades dispares y singularidades preindividuales- se configura, así, como una dramatización que ya no se refiere a nada más que a sus propios movimientos, a sus propias composiciones.

Es interesante notar que en los momentos en los que Deleuze apoya la hipótesis de la suficiencia de lo virtual, de lo virtual como estructura, reintroduce la autonomía de un sujeto de producción. En su ensayo de 1956 sobre Bergson (Deleuze, 2005b), lo virtual es representado, de manera sintomática y polémica, y un poco perversa, como el programa de Hegel realizado: "la Duración o la tendencia es la diferencia consigo misma, y aquello que difiere de sí mismo es inmediatamente la unidad de la sustancia y el sujeto" (p. 52) (15 $^{15}$ Y en su artículo sobre el estructuralismo, Deleuze (2005f) escribe: "el verdadero sujeto es la estructura misma: lo diferencial y lo singular, las relaciones diferenciales y los puntos singulares, la determinación recíproca y la determinación completa" (p. 232) ${ }^{16}$. Pero ¿es realmente adecuado, dada la determinación de lo preindivi-

final constituyen la verdadera instancia de determinación. De acuerdo con este libro, entonces, "el dinamismo comprende su propia potencia de determinar el espacio y el tiempo, ya que encarna inmediatamente las relaciones diferenciales, las singularidades y las progresividades inmanentes a la idea" (p. 328; énfasis añadido). Mil mesetas puede ser comprendido, en buena medida, como el intento de emancipar el poder de este dinamismo de su función como mediación de una encarnación que en últimas está determinada por la Idea, por la suficiencia de la Idea.

15 Hace eco a esta posición, que puede encontrarse en "L'actuel et le virtuel", uno de los últimos trabajos de Deleuze (1995): "la actualización pertenece a lo virtual. La actualización de lo virtual es la singularidad, mientras que lo actual mismo es la individualidad constituida. Lo actual cae del plano [de inmanencia] como un fruto, mientras que la actualización lo relaciona con el plano como lo que convierte de nuevo el objeto en un sujeto" (p. 181; traducción de Juan F. Mejía M.).

16 En este texto, así como en su reseña de Lógica y existencia de Jean Hyppolite (Deleuze 2005a), en donde el programa de la ontología de la diferencia aparece en la forma de "Hegel menos la contradicción”, podemos encontrar algún apoyo para la irreverente evaluación de Badiou (2002), que se apoyaba en el papel de la memoria y el tiempo en Hegel y Deleuze: "la querella entre Deleuze y Hegel pasa por la naturaleza de las operaciones (lo negativo contra lo expresivo, la dialéctica contra la intuición, el despliegue ascendente contra la 'anarquía coronada'), pero no por el montaje 
dual como multiplicidad que es en sí misma disimétrica, y por lo tanto no autónoma, postular una subjetividad originaria (aunque sea una exclusivamente inhumana) en el corazón de los procesos de individuación? ¿Es este sujeto inhumano, bien sea tendencia o estructura, realmente la razón suficiente del devenir? A pesar de las afirmaciones que acabo de citar, pienso que la respuesta es no. La razón puede encontrarse una vez más en el ensayo sobre el estructuralismo. En su conclusión, Deleuze (2005f) se mueve más allá de su ecuación inicial de la estructura y el sujeto, ofreciéndonos una articulación mucho más sutil de los dos términos:

El estructuralismo no es un pensamiento que suprima el sujeto, sino que lo desmenuza y distribuye sistemáticamente, que cuestiona la identidad del sujeto, que la disipa y la desplaza de los lugares sucesivos, un sujeto siempre nómada, hecho de individuaciones, pero impersonales, o de singularidades, pero pre-individuales (p. 247).

Así, nos enfrentamos a la triple articulación de estructura, sujeto y objeto paradójico. Desde esta perspectiva, el último ya no es el único principio autónomo de la heterogénesis. Exige ser acompañado o doblado por un sujeto. Podemos ver así que, lejos de postular simplemente la autonomía de un régimen virtual, esta interpolación de la subjetividad está relacionada con la necesidad de una dramatización sin la cual la diferencia interna de la multiplicidad ideal podría, en el análisis final, ser estéril. En otras palabras, los eventos ideales que pueblan la estructura, como muchas singularidades preindividuales, son dobladas por un evento subjetivo que da cuenta de la explosión de una estructura y la determinación de otra. Aunque en un primer momento el lugar vacío u objeto paradójico parece suficiente para explicar mutaciones estructurales, creemos estar justificados al ver esta introducción del sujeto como indicación de la necesidad de la dramatización, limitando la suficiencia putativa de lo virtual. Deleuze llama a esto la cuestión de la praxis, y junto con la subjetividad, echa una mirada a los criterios del futuro, criterios que, podemos sostener, llevará como aporte a su trabajo con Guattari, así como a la ética. Desde el punto de vista estrictamente ontológico,

global” (p. 93). Por supuesto, la querella sobre las operaciones es, tal vez, la querella filosófica por antonomasia cuando se trata de la cuestión de la individuación. 
o mejor, ontogenético de nuestra investigación, es importante reflexionar sobre esta cuestión de la praxis como el "punto de mutación" que nos permite pensar el proceso de individuación como potencialmente transformativo de la idealidad misma; desplazando las constricciones de la actualización y dando así lugar a una crítica real de la máquina fantasma-en-la-máquina, que pertenece todavía al estructuralismo. Podemos sostener que semejante foco sobre la dramatización subjetiva, del individuo como pensador, ofrece una vía privilegiada hacia la siguiente pregunta: ¿cómo se individúan las propias multiplicidades ideales?, o ¿hay una génesis de la estructura? ${ }^{17}$

\section{Referencias}

Badiou, A. (2002). Deleuze, el clamor del ser. Buenos Aires: Manantial.

DeLanda, M. (2002). Intensive Science and Virtual Philosophy. London: Continuum Books.

Deleuze, G. (1995). L'actuel et le virtuel. En: G. Deleuze y C. Parnet. Dialogues (pp. 179-185). París: Flammarion.

Deleuze, G. (2002a). Diferencia y repetición. (Trad. M. S. Delpy y Hugo). Buenos Aires: Amorrortu.

Deleuze, G. (2002b). Francis Bacon: lógica de la sensación. (Trad. I. Herrera). Madrid: Arena Libros.

Deleuze, G. (2005a). Jean Hyppolite, Lógica y existencia. En: D. Lapoujade (Ed.), La isla desierta y otros textos. Textos y entrevistas 1953-1974 (pp. 21-27). (Trad. J. L. Pardo). Valencia: Pretextos.

Deleuze, G. (2005b). La concepción de la diferencia en Bergson. En: D. Lapoujade (Ed.), La isla desierta y otros textos. Textos y entrevistas 1953-1974 (pp. 45-70). (Trad. J. L. Pardo). Valencia: Pretextos.

Deleuze, G. (2005c). El método de la dramatización. En: D. Lapoujade (Ed.), La isla desierta y otros textos. Textos y entrevistas 1953-1974 (pp. 127-153). (Trad. J. L. Pardo). Valencia: Pretextos.

17 Sobre estos criterios de subjetividad y praxis como límites (edges) del estructuralismo, véase Deleuze, 2005f, pp. 246-249. 
Deleuze, G. (2005d). Mística y masoquismo. En: D. Lapoujade (Ed.), La isla desierta y otros textos. Textos y entrevistas 1953-1974 (pp. 171-176). (Trad. J. L. Pardo). Valencia: Pretextos.

Deleuze, G. (2005e). Hume. En: D. Lapoujade (Ed.), La isla desierta y otros textos. Textos y entrevistas 1953-1974 (pp. 213-222). (Trad. J. L. Pardo). Valencia: Pretextos.

Deleuze, G. (2005f). ¿Cómo reconocer el estructuralismo? En: D. Lapoujade (Ed.), La isla desierta y otros textos. Textos y entrevistas 1953-1974 (pp. 223250). (Trad. J. L. Pardo). Valencia: Pretextos.

Deleuze, G. (2005g). Lógica del sentido. (Trad. M. Moreira). Bercelona: Paidós.

Deleuze, G. (2006). Clase X. El Gran Diádoco. Whitehead y el acontecimiento, 10 de marzo de 1987. En: Exasperación de la filosofía. El Leibniz de Deleuze (pp. 247-262). Buenos Aires: Cactus.

Deleuze, G., \& Guattari, F. (2002). Mil mesetas. (Trad. J. Pérez Vázquez). Valencia: Pre-Textos.

Paradis, B. (1995). Schémas du temps et philosophie transcendantale. Philosophie, 47, 10-27.

Piaget, J. (1968). Le structuralisme. París: Presses Universitaires de France.

Schürmann, R. (1990). Heidegger on Being and Acting: From Principles to Anarchy. Bloomington: Indiana University Press.

Simont, J. (2002). Critique de la représentation et ontologie chez Badiou et Deleuze. En: C. Ramond (Ed.), Alain Badiou: Penser le multiple (pp. 457476). Paris: L'Harmattan.

Toscano, A. (2006). The Theatre of Production. Philosophy and Individuation between Kant and Deleuze. New York: Palgrave.

Vuillemin, J. (1962). La philosophie de l'algèbre. París: Presses universitaires de France. 